\title{
6
}

\section{A VUELTAS CON LA SOCIEDAD DE LA INFORMACIÓN: LA TRANSFORMACIÓN DIGITAL COMO «INNOVACIÓN POSIBLE» PARA ESPAÑA}

El artículo constituye una reflexión sobre algunos de los retos y de las oportunidades que la transformación digital supone para España. En primer lugar, se pasa revista a estudios e índices de referencia internacional en la materia para contextualizar así el reparto emergente de trabajo que gira en Europa alrededor de agendas y agencias, responsables respectivamente de estrategias y programas. En segundo lugar, se identifica la oportunidad de poner en marcha una red internacional de agencias nacionales de transformación digital capaces de activar ecosistemas digitales 4.0 que contribuyan conjuntamente a acelerar el actual ritmo de avance, algo particularmente urgente en el ámbito de la Unión Europa. Por último, entendiendo la transformación digital como innovación y cambio, se analiza el papel de los espacios de cocreación como palancas para sacar el máximo de innovación y la creatividad de las organizaciones a través de sus propios activos y socios potenciales.

Palabras clave: transformación digital, agendas digitales, ecosistemas digitales, agencias nacionales, espacios de cocreación.

Clasificación JEL: O33, O38.

\section{Introducción}

Los creadores del término «transformación digital» en 2011, en el seno del MIT Center for Digital Business, hicieron notar la diferencia sustancial entre «digitalización» y «transformación

\footnotetext{
* Exdirector del Instituto Europeo de Innovación y Tecnología. Ex Director General de Red.es

Versión de octubre de 2018.

Esta publicación no hubiera sido posible sin el apoyo de dos de mis colaboradores más estrechos en la Unidad de Apoyo de la Dirección General de Red.es: Victor Rodrigo y Jorge Rojo a quienes agradezco su inestimable guía y colaboración durante mi mandato. Habiendo sido tan afortunado por la amistad que construimos en mi etapa como director general de Red.es, sirvan estas líneas como muestra de sincera y profunda admiración.
}

digital» (Westerman et al., 2011). Ambos términos están relacionados, pero no son lo mismo porque la transformación digital se apoya en el uso de la tecnología, pero se orienta a «mejorar radicalmente el rendimiento y el alcance de las empresas", lo que lo convierte en un objetivo estratégico para operar un cambio cultural. No en vano algunos autores señalan que transformación es la suma de la innovación y la gestión del cambio (Marcet, 2015).

De manera que la tecnología y los equipos técnicos son necesarios, pero transformar las relaciones con los clientes, la operativa interna de las empresas y desarrollar nuevas $D$ 
propuestas de generación y captación de valor exige mucho más. Al tiempo, las tecnologías digitales tienen un potencial innovador, agilidad y vigencia indudables; baste pensar en las redes sociales, internet móvil, la analítica de datos, el internet de las cosas, etcétera. Este artículo postula la transformación digital como una forma de «innovación posible» para España porque, al contrario que en otros sectores intensivos en capital, «la digitalización va de personas» (Alba, 2016). Y que muchas cosas son posibles y consisten sencillamente en planteárselas.

\subsection{La digitalización, condición necesaria pero no suficiente}

Ya en los primeros trabajos del MIT, y en los numerosos estudios que se han venido desarrollando desde entonces, se encuentra que del extenso conjunto de empresas estudiadas, solo aquellas en las que se unía el compromiso del equipo de gestión en la transformación de la compañía con el uso intensivo de las tecnologías digitales consiguieron aumentar simultáneamente sus ingresos, sus beneficios y su valoración bursátil. Por el contrario, aquellas con un elevado grado de digitalización, pero poco compromiso del equipo de gestión, aumentaron sus ingresos, pero disminuyeron sus beneficios y su valoración bursátil. En el lado opuesto a estas últimas se encuentran las empresas con un equipo de gestión potente comprometido con la transformación de la empresa, pero que realizan pocas inversiones en tecnologías digitales. Estas aumentaron sus beneficios y su valoración bursátil, pero a costa de perder mercado, disminuyendo sus ingresos. Por último, las que no disponían ni de equipos de gestión comprometidos con la transformación, ni invertían en digitalización, empeoraron en todos los

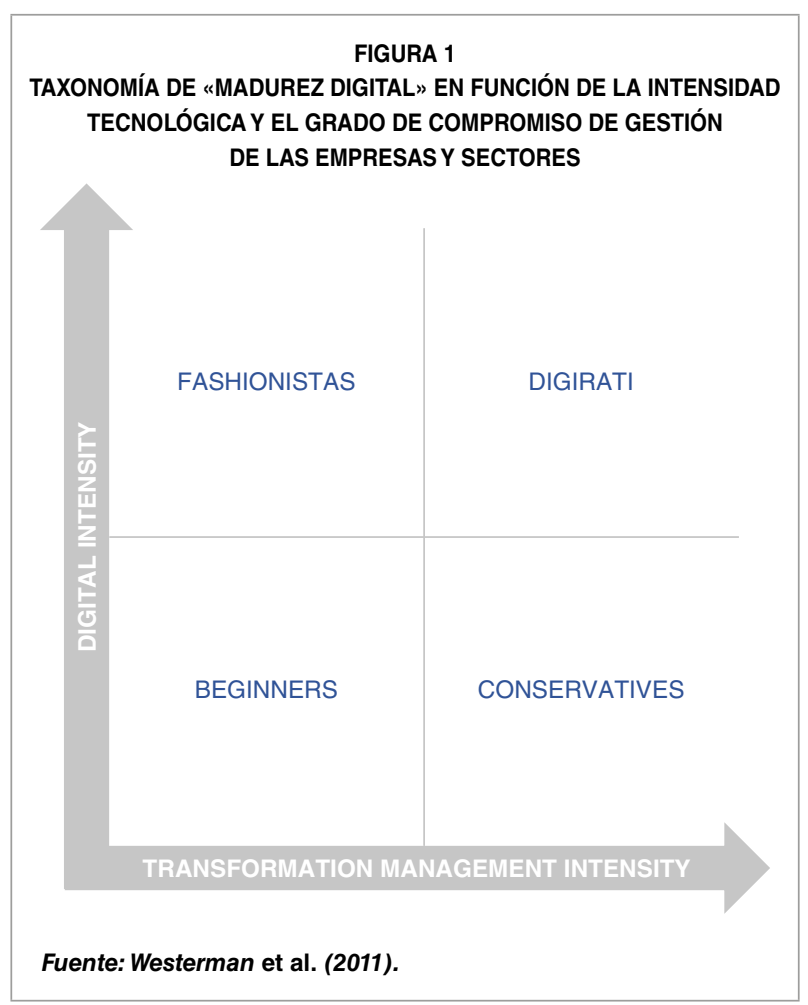

aspectos: ingresos, beneficios y valoración bursátil. En este escenario, ¿cómo saber si una empresa avanza o no en su transformación digital y qué ha de hacer para conseguirlo?

El valor de conocer el grado de digitalización de las empresas reside en que la digitalización es una condición necesaria para avanzar hacia la transformación digital, y que no se puede producir transformación digital sin digitalización. En consecuencia, conociendo el grado de digitalización de las empresas, se conoce qué empresas, potencialmente, pueden aspirar a transformarse digitalmente. Es esta medida, la del grado de digitalización, la que suelen proporcionar organismos nacionales e internacionales. En España, el INE desarrolla una encuesta de uso de TIC (tecnologías de información y comunicación) por parte de las empresas; Red.es publica anualmente un estudio del ONTSI (Observatorio Nacional de las Telecomunicaciones y de la Sociedad de la Información) sobre e-Pyme, en el que $\triangleright$ 
profundizan diversas asociaciones empresariales. En el ámbito internacional destaca, no obstante su carácter macro, el papel de la Comisión Europea, con su índice sobre Economía y Sociedad Digital (DESI) y más específicamente por su subíndice «Integración de la Tecnología Digital en las empresas»; y la OCDE, en sus estudios sobre transformación digital.

\subsection{La oportunidad de la transformación digital en España}

La revisión de los estudios e índices apuntados más arriba indica un desarrollo heterogéneo de la digitalización en las empresas españolas y europeas, con unos sectores mucho más digitalizados que otros y con un fuerte sesgo a favor de las empresas grandes frente a las de menor tamaño. En consecuencia, es de vital importancia impulsar la digitalización de las empresas españolas, especialmente las pymes, colaborando con los equipos de gestión de estas empresas para avanzar desde la digitalización a la transformación digital. Obviamente, dado que no será posible resolver fallos de mercado con carácter general, resulta necesario, por una parte, un cierto ingenio en el diseño de las intervenciones públicas alimentando efectos de emulación a través de casos de uso y, por otra, reforzando la oferta de soluciones nacionales.

Red.es, entidad pública actualmente adscrita al Ministerio de Economía y Empresa a través de la Secretaria de Estado para el Avance Digital, lleva dieciséis años trabajando en la promoción de la Sociedad de la Información con iniciativas y programas en ámbitos muy diversos. No obstante su carácter público, la entidad no renuncia a ser empresarial ni tampoco emprendedora. Partiendo de un foco inicial en
Red.es por la digitalización de servicios públicos con relación a la AGE, CCAA y EELL, son tres los grupos de interés a los que se orienta, además de las Administraciones Públicas, las empresas y, en última instancia, los ciudadanos.

Durante el periodo citado se ha lanzado un amplio abanico de iniciativas: programas formativos para desarrollar profesionales digitales, comercio electrónico, emprendimiento y en la internacionalización de las empresas; impulso a la adopción de soluciones tecnológicas, como el de cloud computing, etcétera, difundiendo las soluciones tecnológicas para pymes y autónomos, y apoyando a sectores específicos claves para la economía y la sociedad, como la sanidad, la educación o el turismo.

Con la aprobación del Plan Estratégico 2017-2020 la transformación digital pasa a ser el leitmotiv de Red.es, que es la entidad de la Administración que tiene encomendada formalmente esta misión. De tal modo, la entidad se prepara para la futura estrategia digital que tenga a bien decidir el Gobierno. Y si en materia de servicios públicos digitales España está ya en posiciones avanzadas, comparada con otros países de nuestro entorno, ha de profundizarse aún más en lo relativo a economía y sociedad digitales. Efectivamente, las empresas y los emprendedores son el corazón de la transformación digital. Los programas futuros deben partir del convencimiento y del compromiso de sus gestores con la utilidad de la transformación digital, para lo cual la entidad ha de abrirse a sectores existentes y conectar asimismo con ecosistemas emergentes.

Por todo ello, Red.es puede y debe ser el «punto de encuentro» para auspiciar este tipo de dinámicas, porque la innovación no se decreta, y para que la entidad tenga mayores garantías de acierto e impacto aspira a ser cauce de los objetivos de los agentes recogiendo $\triangleright$ 
necesidades y diseñando iniciativas de interés general. En esta línea, en 2017 se puso en marcha el Foro Red.es, un punto de encuentro para confrontar y cocrear iniciativas futuras en consulta a todos los grupos de interés: «rediseñar lo que somos para transformar lo que hacemos", como reza el Plan Estratégico. En 2018, se pusieron en marcha dos nuevos formatos: Ágora y el Campus Red.es, que reunieron en la Universidad Internacional Menéndez Pelayo de Santander, por segundo año consecutivo, a todos los responsables digitales de las CCAA y la OCDE, impulsando, a la vez, la cooperación interterritorial y el aprendizaje internacional de buenas prácticas.

La aprobación del citado plan supuso un hito para la entidad. Desde el registro español de Dominios.es, que explica el acrónimo que da nombre a la entidad, Red.es se plantea ahora ser «referente excelente digital» tanto nacional como internacionalmente. Con el apoyo de todos, Red.es abrió una nueva etapa. Una etapa en la que se convocó a todos a participar para relanzar y reposicionar una entidad aún más alineada con los tiempos, porque la transformación es precisamente un tema cuyo tiempo ha llegado.

\subsection{Tendencias en Europa: la división natural del trabajo entre agendas $y$ agencias}

La transformación digital se extiende a todas las áreas de la economía y la sociedad, y no comprende fronteras nacionales o sectores específicos. Afecta a empresas y personas, pero cada vez más a nuestras vidas y al funcionamiento de nuestras organizaciones. Por todo ello, un número creciente de Gobiernos estima, llegado el momento, reflexionar sobre los potenciales beneficios, pero también hacer frente a los indudables desafíos que plantea la transformación digital, particularmente en Europa si el viejo continente quiere desarrollar su particular modelo y desempeñar su papel en la era digital o «tercera ola» (Case, 2016). La revisión intermedia del Mercado Único Digital publicada por la Comisión Europea en mayo de 2017 destacó tres áreas en las que Europa debe focalizarse a futuro: la economía del dato, las plataformas digitales y la ciberseguridad, bien entendido que no todo podrá hacerse al nivel de la Unión.

Para avanzar más rápido, experimentar y escalar las políticas más adecuadas, al igual que sucedió con las políticas de innovación en los años 1990 tras la publicación del famoso Libro Verde, algunos países avanzan hacia una división natural del trabajo para fomentar a la vez la reflexión y la acción sostenida en el ámbito de la transformación digital. Tal es el caso de Francia, a cuyo Consejo Digital (Conseil du Numerique, CNNum) corresponde la creación de una función de reflexión, inteligencia y prospectiva independiente, y que en España correspondería al Observatorio Nacional de Telecomunicaciones y Sociedad de la Información. Paralelamente, empiezan a proliferar estructuras dedicadas a la acción, como la Agencia Digital francesa (Agence du Numerique, AdNumm), que hacen pensar que no estará lejos el día en que pueda plantearse una red transnacional de agencias nacionales enfocadas específicamente a acelerar la transformación digital en una lógica de «abajo arriba» y aprendizaje mutuo. Esto es así, ya que muchas cuestiones no se podrán resolver desde Bruselas, cuyas instituciones se centran naturalmente en la regulación y no podrán avanzar rápido sin las actuaciones de los países miembros sobre el terreno, experimentando y $\triangleright$ 
FIGURA 2

ARQUITECTURA DE MODERNIZACIÓN, ASESORAMIENTOY PROMOCIÓN DIGITAL EN FRANCIA

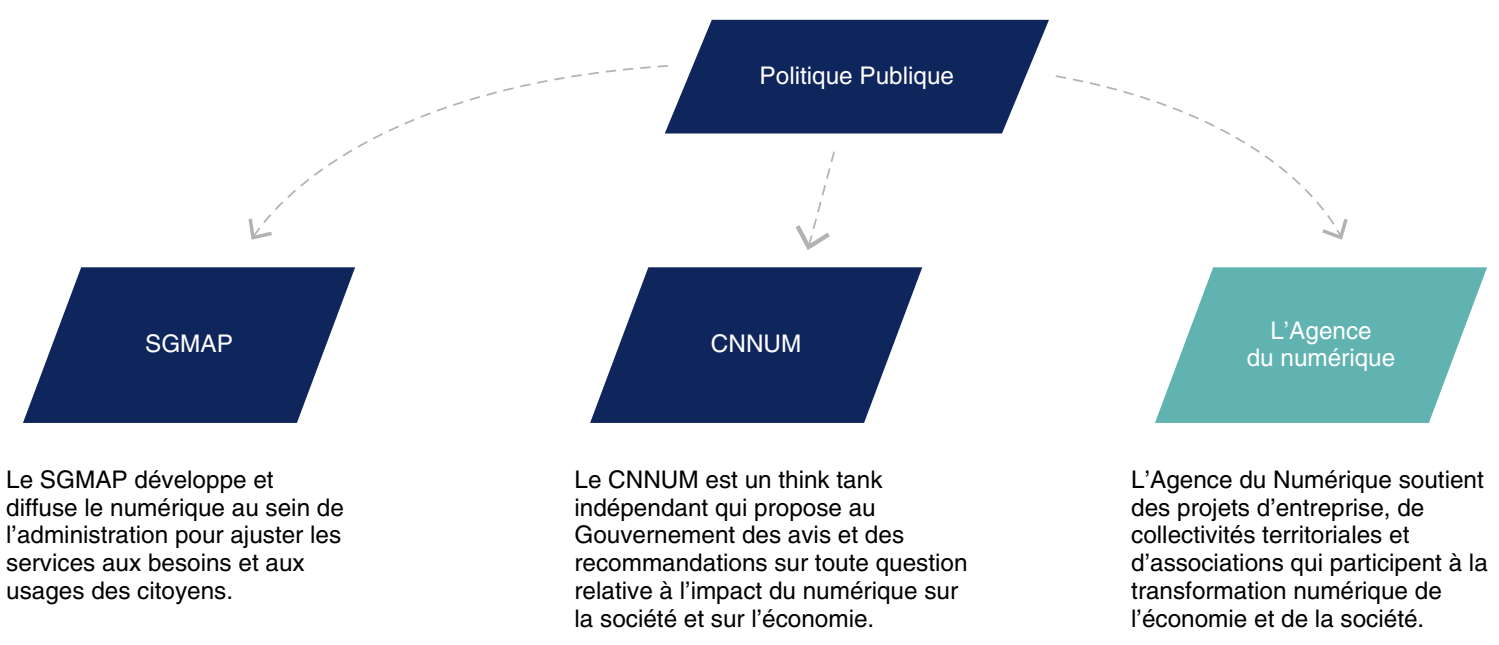

Fuente: https://agencedunumerique.gouv.frl

escalando las mejores prácticas de cada agencia. Curiosamente, aunque cada vez más países están creando agencias digitales, la entidad pública Red.es es quizás una de las entidades nacionales más veteranas de Europa desde su creación en $2002^{1}$.

\subsubsection{Un marco europeo de reflexión} de arriba abajo: hacia una función de inteligencia colectiva y prospectiva independiente a nivel de la Unión Europea

Decidir cuáles son las acciones públicas a implementar y, en su caso, qué iniciativas abordar, requiere una capacidad analítica y prospectiva independiente que tenga en cuenta el interés general, las tendencias tecnológicas y las preferencias de los ciudadanos europeos.

\footnotetext{
1 Italia dispone de una Agencia Digital que reporta al presidente del Consejo de Ministros (https://www.agid.gov.it/en), que ha complementado desde 2016 con un equipo del comisario para la Agenda Digital (https:// teamdigitale.governo.it/en/). Alemania incluyó en la revisión de su Agenda Digital la puesta en marcha de una agencia especializada. Suecia ha dado los primeros pasos con la creación de una Agencia Digital cuyas operaciones se han iniciado el 1 de septiembre de 2018 (https://www.digg.se/about-us).
}

Efectivamente, no se trata simplemente de extender las acciones emprendidas en otras partes del mundo, de las que ya se hacen eco con frecuencia trabajos de consultoría auspiciados por grandes corporaciones. Por tanto, es preciso analizar el impacto que estas transformaciones podrían tener sobre la economía y la sociedad en Europa para determinar con independencia cómo maximizar el impacto y resolver problemas comunes para el interés general del conjunto de la Unión.

Además, la naturaleza local de los problemas, junto con los flujos transfronterizos de conocimiento, exige a los miembros de la UE obtener una visión compartida de las cuestiones que deben abordarse y de las soluciones que deben adoptarse. Esta tensión local-global caracteriza al mundo digital. Las entidades públicas de los Estados miembros han reflexionado individualmente sobre algunos de estos problemas. Los rápidos cambios, sin embargo, invitan a compartir visiones porque Europa no dispone hasta la fecha de un think tank europeo específicamente digital, con recursos e independencia suficientes. La puesta en común de $\triangleright$ 
los órganos de reflexión especializados existentes en varios países permitiría formular propuestas de soluciones más inteligentes y ágiles.

En este sentido, el CNNum francés puso en marcha un piloto a finales de 2017 consistente en un conjunto de grupos de reflexión basado en las capacidades disponibles en los Estados miembros (think tanks nacionales en particular) para generar consenso sobre los temas y nichos de oportunidad que deben abordarse en el futuro. La propia Comisión Europea animó estas reflexiones en la Cumbre de Tallin, que tuvo lugar a finales de septiembre de 2017, y que abordó la transformación digital por primera vez a nivel de los jefes de Estado y de Gobierno.

\subsubsection{Un marco internacional para activar} ecosistemas digitales de abajo arriba: hacia una red internacional de agencias nacionales

Paralelamente a la puesta a disposición del marco de reflexión indicado más arriba, el despliegue de los efectos beneficiosos de la transformación digital requiere también un marco de acción (Do Tank) para que Europa pueda centrarse en las áreas con potencial y acelerar el progreso al federar esfuerzos paralelos a los estímulos reguladores de los organismos europeos y de los Estados. Las instituciones de la UE y de los Estados miembros desarrollan continuamente estrategias y planes de acción digitales para favorecer la transformación digital de la economía y la sociedad, pero concertar las actuaciones de abajo arriba no es evidente en geometría variable.

Frente a cuestiones que pueden ser promulgadas por directivas con éxito visible, como la itinerancia o portabilidad, el éxito de Europa en la transformación digital requiere la activación de ecosistemas digitales subnacionales con ambición internacional. Un ejemplo claro son las Smart Cities, que, según muchos expertos, son lo más cercano a los laboratorios de innovación digital. Por todo ello, el diseño e implementación de estrategias digitales (agendas) dependerá cada vez más de estructuras especializadas (agencias) en un número creciente de países.

La naturaleza heterogénea de tales estrategias y estructuras tiene un valor para compartir conocimientos y experiencias, distinguiendo el nivel de las estrategias y políticas aguas arriba (gobernanza) del de la gestión especializada y experimentación (propia de las agencias), frente a la evaluación y seguimiento aguas abajo (que ha de ser independiente por definición). Estos tres planos son precisamente los que se distinguen claramente en las misiones que prepara la Unión Europea en el contexto del próximo Programa Marco (Figura 3) y que están presentes en mayor o menor medida en la dirección, ejecución y monitorización de las agendas digitales que conocemos.

Animado por la propia Comisión Europea, Red.es explora desde 2017 la creación de una red de agencias abierta a todos los agentes nacionales públicos de toda Europa y, eventualmente, de otras geografías, como agentes responsables de la configuración o implementación de programas digitales, creando así un cuerpo de conocimientos e intercambio de experiencia que permita sacar el máximo partido del conjunto. Como en el caso de la innovación, la red TAFTIE (www.taftie.org) alentaría a un número creciente de países a incorporar estructuras profesionales y estables a sus acciones públicas en el campo digital. Dicha red cumpliría varias funciones:

- Base de conocimiento: compartir conocimientos y experiencia en el diseño y $\triangleright$ 


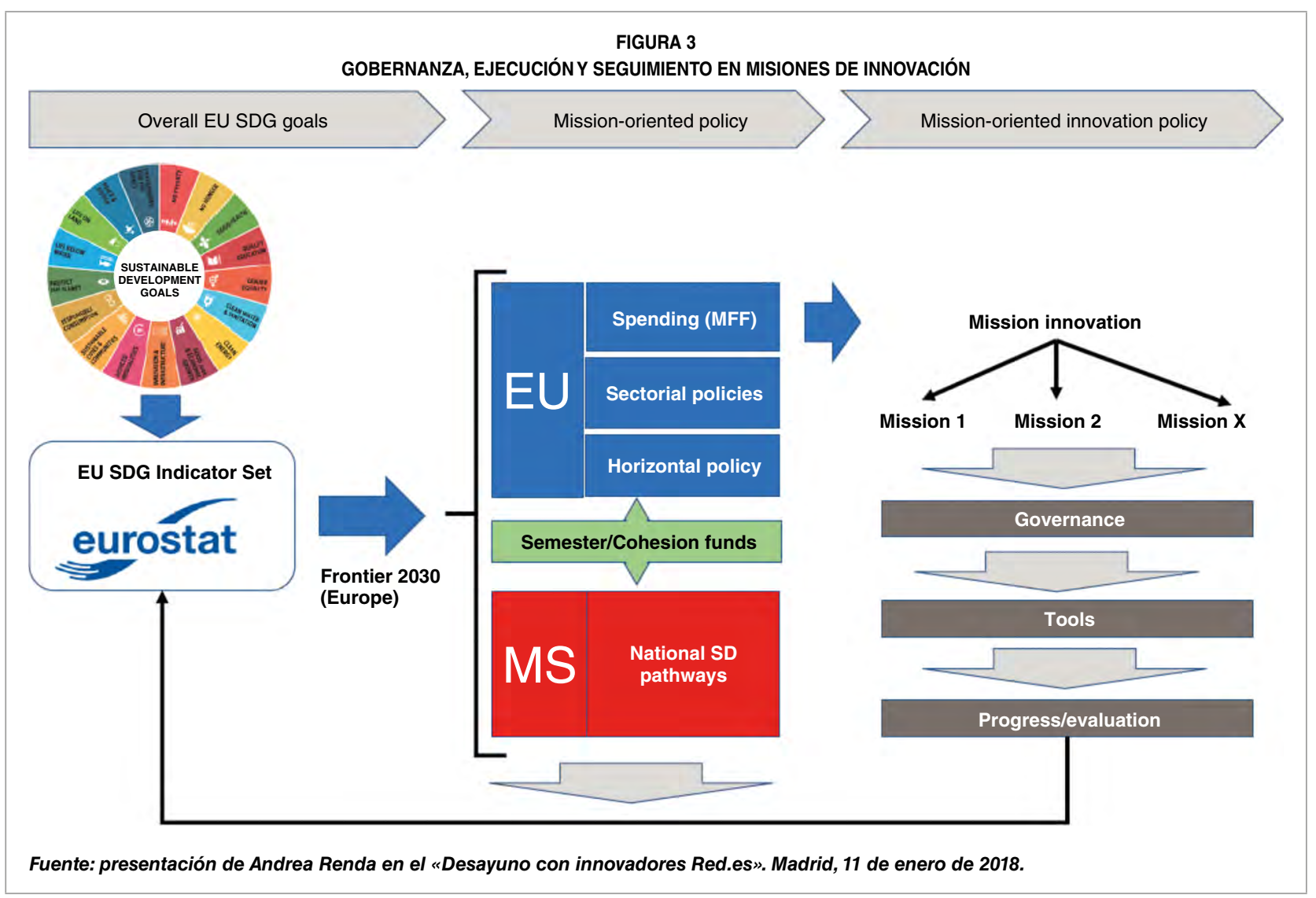

ejecución de políticas digitales orientadas a lograr la transformación digital de la sociedad, la economía y las Administraciones Públicas.

- Academia en red: crear una comunicación estable y mecanismos de apoyo mutuo entre los miembros y sus grupos de interés facilitando futuros proyectos conjuntos.

- Foro sobre políticas: profundizar en la cooperación entre los servicios de estudios respectivos y las autoridades de tutela correspondientes.

Los miembros de la red deberían ser entidades legales y tener capacidad de gestión independiente para contribuir a la independencia requerida de la red como un todo. La red tendría una geometría variable, permitiendo que las agencias se incluyan en grupos de trabajo compatibles con su competencia nacional. Durante la fase inicial, a través del ONTSI, Red.es se mostró dispuesto a liderar la creación de un directorio de agentes, gestores y contactos, en forma de repositorio de políticas públicas digitales, que sirviera más adelante como herramienta para difundir las estrategias y prácticas desarrolladas por los Estados miembros de la UE, la identificación de una herramienta para facilitar el contacto entre todos los miembros de la asociación y otras partes interesadas, la creación de un espacio de trabajo colaborativo para los miembros de la red y el diseño de un programa de reuniones en línea o en persona y/o seminarios sobre políticas públicas digitales y su impacto, en colaboración con grupos de interés. 


\section{Espacios de cocreación: GoingDigital, «scale, scope, speed»}

Para activar los tres niveles indicados más arriba (dirección, ejecución y monitorización), habida cuenta del cambio de paradigma que supone la revolución digital, hay que insistir en la importancia de instrumentar una fuerza de experimentación y aprendizaje organizativo. Efectivamente, si la transformación digital se define como innovación y cambio, cualquier organización pública o privada ha de esforzarse por sacar el máximo de innovación y creatividad de sus propios colaboradores. Porque ambas se enfrentan al reto de sacar el máximo partido del talento que las integra y, al tiempo, abrirse a las ideas del entorno. $Y$ para ello tienen que buscar mecanismos con los que desarrollar capacidades intramuros y extramuros que les permitan asegurar su eficiencia y eficacia, al tiempo que crean y renuevan sus propuestas de valor. Una organización creativa e innovadora aporta muchos beneficios, que favorecen las condiciones para su sostenibilidad futura en un mundo donde el cambio se acelera.

Autores como Ramaswamy y Gouillart (2010) describieron cómo algunas empresas se habían desarrollado más rápidamente gracias al uso de técnicas como el crowdsourcing (participación de un extenso grupo de personas, además de los propios empleados y proveedores de una empresa, en las tareas de una organización), la colaboración colectiva y la generación de ideas. Igualmente, otras organizaciones habían desarrollado nuevas formas de interacción con sus trabajadores alcanzando niveles insospechados de actuación a través de la cocreación. De esta forma, los directivos empezaron a abandonar la mentalidad tradicional vinculada a los productos y servicios para adoptar otra muy diferente, basada en la experiencia de usuario.

Muchas empresas cocreativas generan ideas únicas e innovadoras gracias a las experiencias y propuestas de sus grupos de interés, que son quienes verdaderamente participan del servicio o producto final en el propio diseño. Estas organizaciones utilizan entornos de colaboración en los que distintos equipos pueden interrelacionarse consiguiendo optimizar recursos y costes. A todos nos viene a la mente ejemplos de cocreación de organizaciones internacionales, como son la marca de ropa deportiva Nike, las tiendas de ordenadores Apple Retail Stores o la multinacional de juguetes danesa LEGO. En los últimos años se han sumado a esta tendencia importantes compañías españolas como Bankinter, Freixenet, Orbea, Correos, etcétera.

En este nuevo contexto, muchas empresas han comenzado a estimular la participación de su personal. Asimismo, involucran a usuarios como cocreadores activos que participan en la definición de las prestaciones y del valor del producto. La organización cocreativa es un magnífico mecanismo de productividad que busca la calidad, aumentando la participación del empleado en cooperación con los grupos de interés de la organización que participan de uno $u$ otro modo en la cadena de valor del producto o servicio. $Y$ con todo ello, una organización cocreativa se convierte en una fuerza de progreso e innovación que enriquece el capital estratégico y aumenta las oportunidades de la organización y en el sector en que opera.

El proceso de cocreación involucra dos pasos básicos: generación de contribuciones y selección de las mismas. Dependiendo del grado de control de la propia organización (empresa o entidad pública) o el grupo de interés $\triangleright$ 


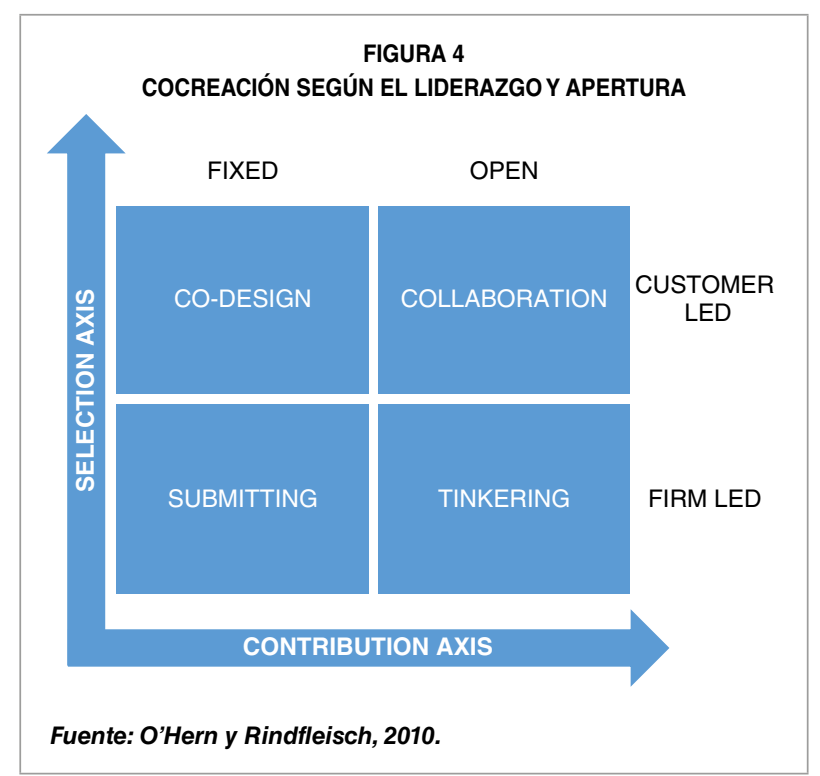

(clientes o público objetivo) en la generación de la selección, la cocreación se puede clasificar en general en cuatro categorías (Figura 4).

Se trata, pues, de una transformación total de la cultura y las capacidades corporativas en diseño, creación y ejecución del valor de las empresas. Y por todo ello las entidades públicas son cada día más conscientes de la necesidad de desarrollar nuevas formas de organización y gestión más innovadoras. La OCDE y NESTA tienen programas en marcha para recoger la experiencia de laboratorios de innovación, y una literatura incipiente presenta evidencias de que el sector público no solo puede fomentar y financiar la innovación que desarrollan empresas y emprendedores, sino que puede y debe hacerlo también revisando la forma en que trabaja (Leyden y Lynk, 2015).

\subsection{La Enredadera. Hacia un espacio de cocreación en Red.es}

Como hemos indicado anteriormente, en la era digital de la nueva economía, innovar es imprescindible no solo para el sector privado, sino también para el público (Daglio et al, 2015). Por otra parte, en la era de los datos que la revista The Economist postuló en una famosa portada de mayo de 2015, «The world's most valuable resource» (https:// www.economist.com/printedition/covers), avanzar hacia la innovación abierta con los datos parece la frontera natural para España, habida cuenta del lugar referencial que ha logrado en el panorama europeo de la apertura de datos públicos. Red.es tiene aquí un punto de arranque para ideas en estado embrionario que podría concretar: acelerar a los aceleradores, aceleradores en residencia, escaladores internacionales, llamadas de oportunidad; términos todos ellos a la búsqueda de oportunidad.

En esta exploración es cierto que las organizaciones del sector público están reguladas por una compleja red de leyes, instrucciones y procedimientos. Estas incluyen limitaciones presupuestarias, procedimientos internos, protocolos de comunicación, marcos legales que regulan sus actividades en áreas como la privacidad, la seguridad o la adquisición de recursos. Sin embargo, la capacidad de entidades públicas para escalar e impactar es incuestionable, particularmente si son capaces de aprender.

Si bien las reglas de buen gobierno se establecen por buenas razones (proteger el interés público, garantizar el uso ético de los recursos, promover la responsabilidad, establecer procedimientos operativos comunes para garantizar la eficiencia y sostenibilidad de las organizaciones), su diseño puede tener efectos involuntarios capaces de inhibir las acciones individuales y organizacionales en la capacidad de innovar y emprender.

A pesar de ello, la Administración cuenta con importantes herramientas con las que $D$ 


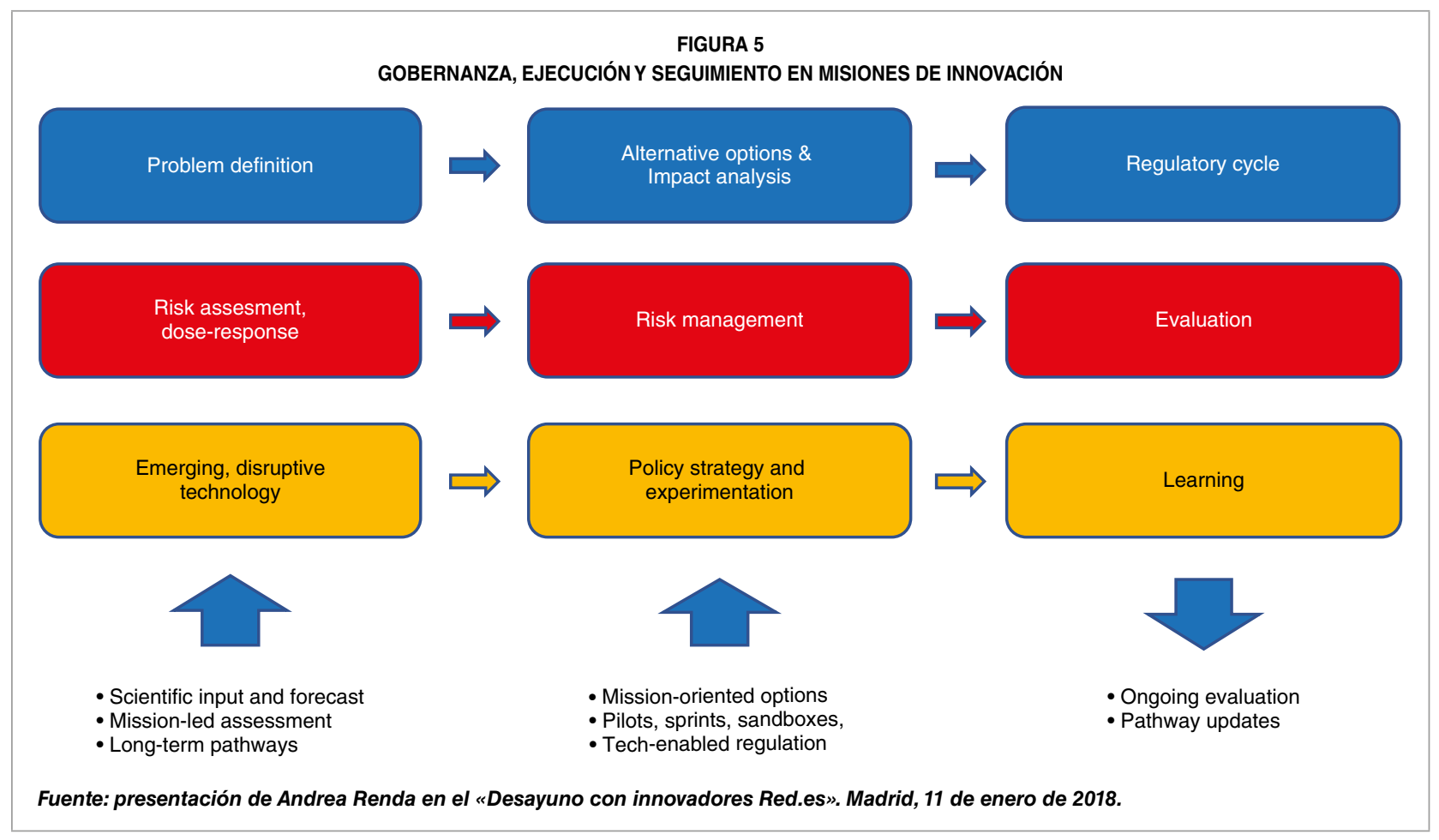

fomentar la innovación y el emprendimiento (Hughes et al, 2009), como:

- Crear nuevos programas y espacios que promuevan la implementación de aplicaciones TIC que permitan la interacción entre ciudadanos y empresas, incluido el desarrollo de nuevas plataformas de TI.

- Eliminar barreras y crear incentivos para que las organizaciones del sector público construyan una cultura innovadora y sean capaces de implementar innovaciones.

- Difundir casos de buenas prácticas innovadoras en el ámbito del sector público.

- Fomentar las colaboraciones públicoprivadas para proporcionar soluciones a la sociedad y los nuevos desafíos económicos.

- Diseñar sistemas de sandbox, con el objetivo de atraer empresas y talento, y reducir las fricciones regulatorias que la innovación y la tecnología encuentran en muchos sectores.

- Facilitar la creación de ecosistemas innovadores.

- Fomentar el desarrollo y la adopción de nuevas tecnologías y métodos de gestión innovadora.

Esta imperiosa necesidad de innovación y emprendimiento en el seno del sector público representa muchos retos, pero también innumerables oportunidades para mejorar la eficiencia de los servicios públicos, la confianza en los Gobiernos y la calidad de las democracias. Para Red.es significaría también la oportunidad de avanzar de manera práctica hacia una forma de trabajo aún más eficaz, aprovechando su singularidad como una de las pocas agencias en España que son, hoy por hoy, capaces de trabajar a la vez la oferta y la demanda, lo que, partiendo de la inteligencia del ONTSI y buenas prácticas internacionales, $D$ 
con autoridades nacionales de tutela, grupos de interés y recursos propios, permitiría delinear programas aún más eficaces y bien diseñados.

En este contexto, como resultado de un concurso de ideas interno, Red.es diseñó e inauguró a finales de 2017 su espacio destinado a la cocreación, denominado La Enredadera, un espacio de más de 200 metros cuadrados que la entidad ha dedicado en primera instancia a activar el talento interno y en segunda instancia a acoger eventos en colaboración con asociaciones y colectivos que integran el ecosistema digital español. Idealmente, La Enredadera nació también con vocación de dedicarse a la cocreación de proyectos futuros como un espacio flexible y adaptable que permite multiplicidad de usos para trabajo en equipos pequeños, encuentros espontáneos, formación, etcétera.

Ubicada en la segunda planta del Edificio Bronce de Madrid, La Enredadera reúne las capacidades para crear, desarrollar y aplicar innovaciones disruptivas y acelerar su proceso de transformación digital. Tras abrirse a los empleados de la entidad, se determinaron las condiciones para poner La Enredadera a disposición de otros organismos públicos, asociaciones y empresas. Porque la entidad entendió que la colaboración e interrelación entre los diferentes agentes económicos y sociales es clave para que los beneficios experimentados por los grandes actores del sector TIC se hagan extensibles también a pymes y autónomos con grados de digitalización menores.

Con todo ello, Red.es vendría a combinar su propia experiencia en transformación digital, la innovación y el conocimiento de sus profesionales con las tecnologías digitales más relevantes de las empresas del sector, sirviendo de cauce para iniciativas que difícilmente saldrían de un despacho. Una de las ventajas de este tipo de espacios en otras organizaciones consiste en que los participantes olvidan su filiación y posición jerárquica, de manera que la competición se produce para dar lo máximo en la generación de nuevas ideas. Por todo ello, la entidad puede conectar con el ecosistema digital a través de La Enredadera incluyendo concursos de ideas para seleccionar las mejores que permitan a la vez cultivar y trabajar en comunidades y grupos de interés buscando efectos de emulación y movilización social.

Durante sus primeros meses de vida en 2017, Red.es reforzó su Plan de Formación incorporando un eje de técnicas de innovación y creatividad, aprovechando el espacio La Enredadera para formar a todos los empleados interesados en la metodología Design Thinking. Esta metodología consiste en la innovación centrada en las personas, ofreciendo una lente a través de la cual se pueden observar los retos, detectar necesidades y formular soluciones mucho más creativas. Otras técnicas seguirán en 2018 (Lean Startup, Blue Oceans, Agile, etcétera).

Esta primera fase de uso interno ha dado paso en 2018 al uso externo de La Enredadera con autoridades, colectivos y agentes externos. En los primeros meses tuvieron lugar sendos seminarios con los Agentes Registradores de Dominios.es, la presentación del nuevo Plan Nacional de Territorios Inteligentes y unas jornadas sobre «El fenómeno de la desinformación en la era digital». Que el espacio sirva de cauce para debates y propuestas tiene un singular valor para la entidad, recordando que fueron los propios empleados quienes diseñaron y dieron nombre a La Enredadera, y viene a subrayar la importancia de la misión que lleva a cabo la entidad. Ello, unido a otras iniciativas para promover el orgullo de pertenencia (15 años de historias de éxito, para el $15 \square$ 
aniversario, por ejemplo), charlas inspiradoras con protagonistas de alto nivel (Desayunos con innovadores) y una información más fluida y estructurada a todos los niveles, ha de sentar las bases para que la entidad pueda seguir liderando con el ejemplo también en la construcción de futuras iniciativas y programas aún más sofisticados.

\section{Conclusión}

El futuro programa marco que sustituirá al actual Horizonte 2020, y que provisionalmente se conoce como «Horizon Europe», avanza en torno a «retos», muchos de los cuales podrían direccionar todo el potencial de las TIC al servicio de misiones. La experiencia de otros países demuestra que, en la puesta en marcha exitosa de tales misiones, el vehículo es tan importante como la idea. Así, por ejemplo, DARPA, la agencia paradigmática de la que surgió Internet, es para muchos, más que una agencia, una cultura en la forma de reclutar el talento y de conectar con el ecosistema.

Desde el discurso de la innovación que apareció en los años noventa en torno al Libro Verde comunitario, un número creciente de países decidieron pasar a la práctica y crear entidades especializadas, agencias que han marcado toda una época, como Tekes en Finlandia o FFG en Austria. De manera análoga, parece claro que tras la etapa de las agendas digitales, observaremos un fenómeno similar, el cual permitirá multiplicar las vías de experimentación, evaluación y aprendizaje comparado de políticas, programas y proyectos a través de agencias digitales nacionales.

Hasta ese momento, las políticas más canónicas de impulso a la innovación se caracterizaban por su linealidad. Con la aparición de nuevas tecnologías habilitadoras y el ritmo del cambio que inducen surge la visión de los ecosistemas. Esta es la «magia» de las TIC, por el triple cambio de registro que supone en escala, profundidad y velocidad del cambio que hacen posible. Con lo digital se ha venido a democratizar la innovación abierta y disruptiva, aunque no esté exenta de retos, ya que «haciendo lo correcto uno puede equivocarse», según recuerda Clayton Christensen en El dilema del innovador, que cumplió en 2017 ya veinte años (Figura 6).

Efectivamente, las TIC lo cambian todo y seguir a los clientes actuales no es necesariamente la receta del éxito. Es la tiranía del Excel y del análisis estrecho de las cuotas de mercado lo que explica alguna de las posiciones que perdió Europa tras la era del puntocom. Pero si la innovación se democratiza, las políticas para la innovación se hacen cada vez más sofisticadas, porque nadie dijo que fueran sencillas. Sabemos, eso sí, que para tener éxito han de ser más globales e integradoras. Frente a las visiones del «sistema de innovación», los ecosistemas ponen el énfasis en las interrelaciones, no intermediadas por instituciones, sino por las personas. Con frecuencia las ideas más rompedoras pueden venir de la periferia. Pero al factor humano hay que sumar el factor social para catalizar el cambio; es preciso que confluyan en un entorno una serie de factores que definen lo que podríamos llamar un «ecosistema 4.0».

Desde la innovación basada en la ciencia, pasando por la colaboración público-privada, donde aún hoy están instaladas la mayor parte de las políticas públicas de apoyo a la innovación empresarial, evolucionamos hacia la experimentación y exploración a través de ecosistemas. Acceso al talento, acceso a la financiación, acceso a mercados, acceso a $\triangleright$ 


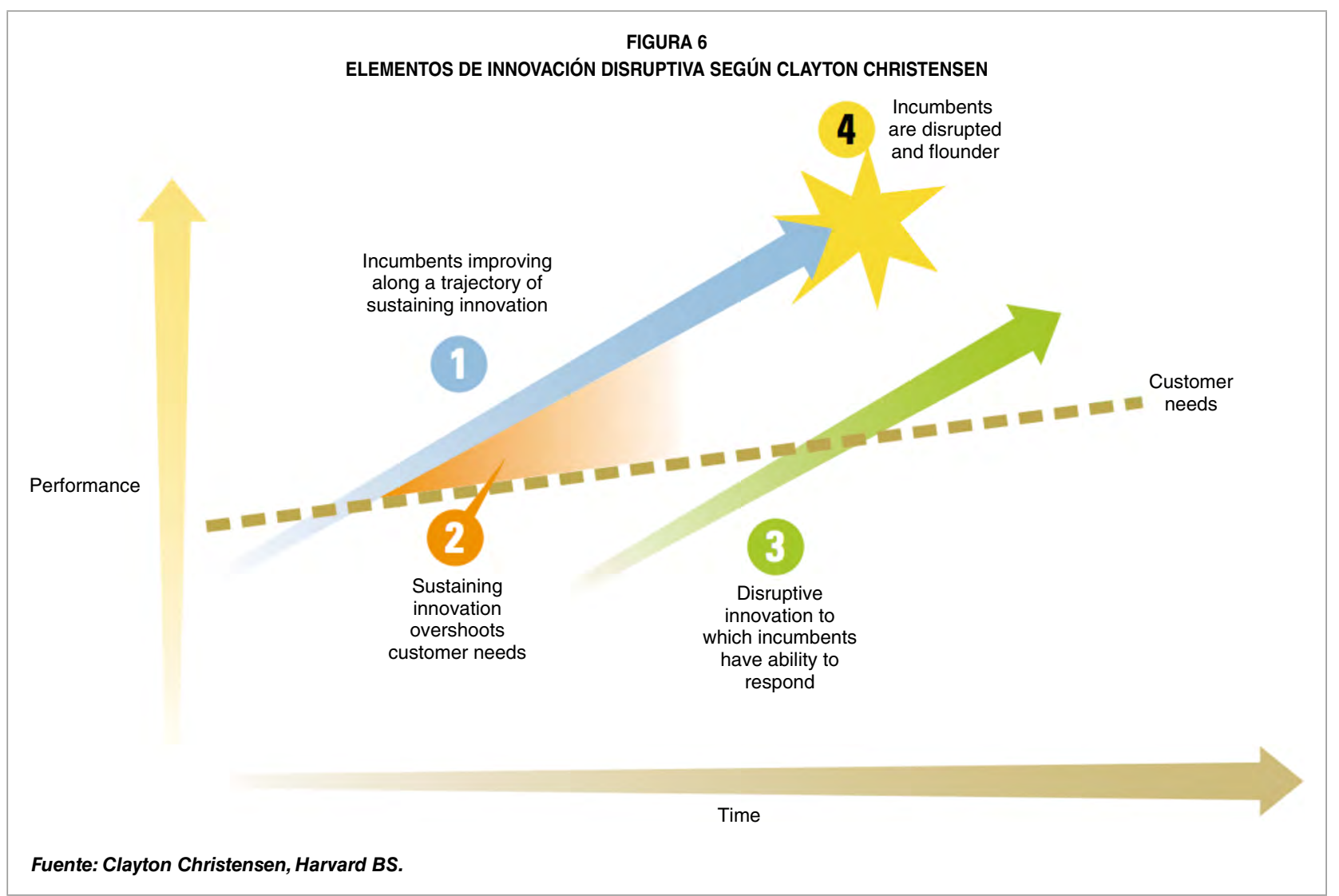

conocimiento, etcétera, que el padre de los ecosistemas emprendedores, Daniel Isenberg, postula en un ecosistema emprendedor de éxito (Figura 7).

Una nueva situación que supone una gran oportunidad para situar a España al nivel de otros países de nuestro entorno más allá de infraestructuras, conectividad o servicios públicos digitales, que, siendo necesarias, no son suficientes. A tal fin, la oportunidad es también un reto no solo tecnológico, ya que cuando hablamos de transformación y ecosistemas, estamos hablando de un profundo cambio cultural. Por tanto, es necesaria la colaboración entre Administraciones, empresas y el resto de los actores económicos y sociales para conseguir que la innovación y lo digital lleguen a todos los ámbitos de nuestra sociedad. Porque la revolución pendiente tiene más que ver con las conciencias que con los laboratorios.
En este sentido, un programa de análisis comparado entre países (agendas gubernamentales) y experiencias organizativas (estructuras dedicadas) tendría el máximo interés para mejorar la comprensión sobre los nuevos roles de las agencias «schumpeterianas» superando las más tradicionales agencias de desarrollo o «weberianas» (Breznitz, 2007; Kattel et al., 2017), y fomentar así las políticas de próxima generación; y en la práctica, para facilitar y organizar la innovación y los ecosistemas emprendedores más adecuados para la transformación digital.

Dicho programa de investigación podría incluir al menos los siguientes contenidos:

- Examinar la evidencia sobre los factores de éxito y fracaso en la experimentación llevada a cabo hasta la fecha en los países de referencia, en el ámbito de las $D$ 


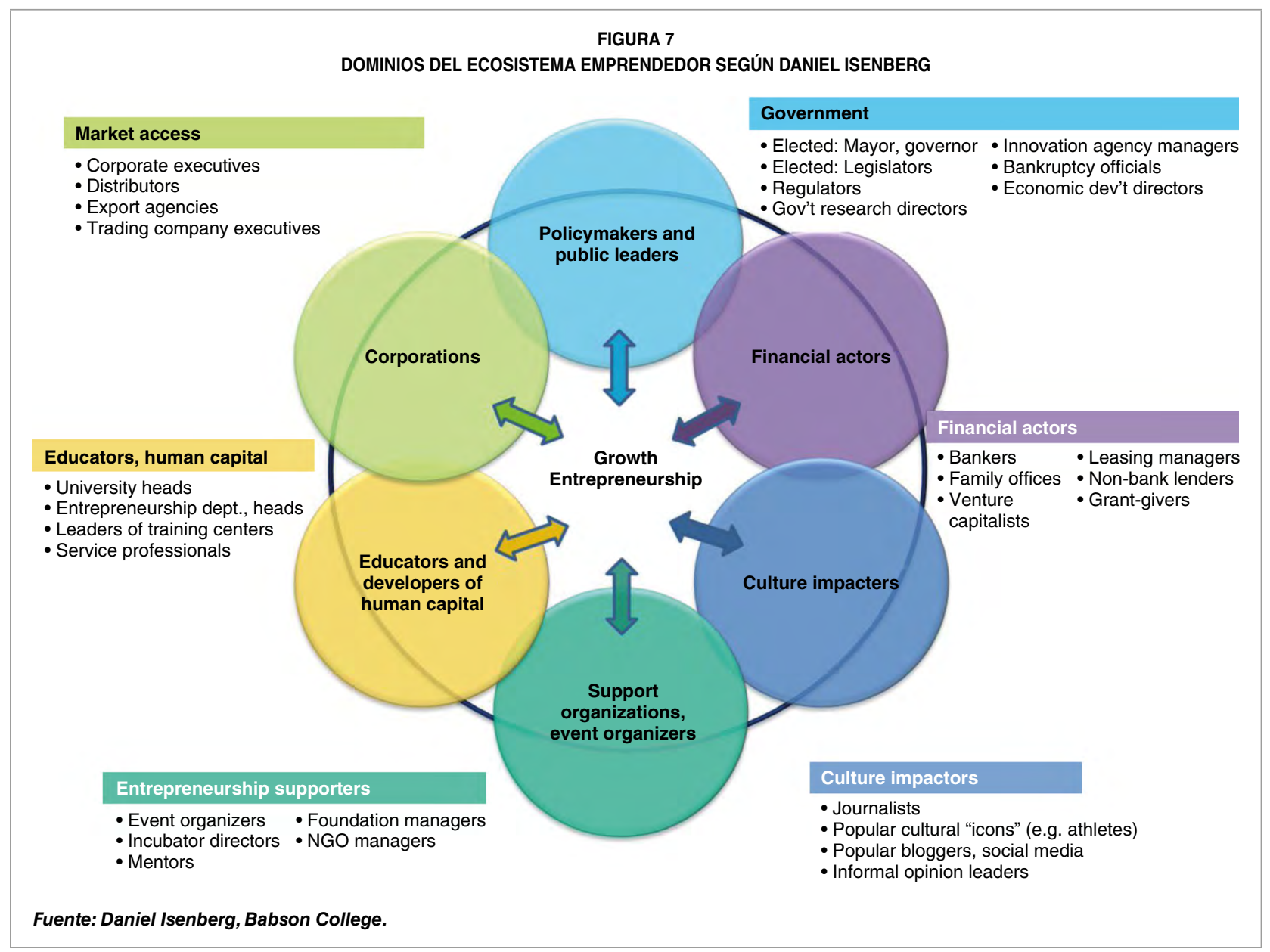

innovaciones y el emprendimiento para la transformación digital.

- Desarrollar los modelos conceptuales, gobernanza y gestión necesarios para una mayor experimentación en políticas a través de la evaluación y aprendizaje.

- Participar y recoger la investigación para la acción en la experimentación en curso que vincula agencias de innovación y ecosistemas emprendedores, y estudiar las correlaciones efectivas entre agencias y ecosistemas digitales.

Efectivamente, en la búsqueda de los esquemas más efectivos, el aprendizaje es clave no solo para implementar programas, sino para dar forma a intervenciones eficientes y responsables. El Parlamento Europeo dispone de un mecanismo de asesoramiento, y también el Congreso de EEUU se ha interesado por la investigación comparada con otros países y la evaluación de las agencias existentes para poner en práctica políticas más exitosas y holísticas, antecedentes que quizá puedan inspirar a los grupos y comisiones de trabajo del Congreso de los Diputados.

Del análisis de los medios necesarios para acelerar el paso en la apuesta por la transformación digital que el conjunto de la Union Europea (Think and Do Tanks) precisa se extrae una conclusión posibilista para el caso de España, en la medida en que nuestro país es privilegiado para abordar efectivamente políticas 4.0. de transformación digital al $\triangleright$ 
disponer no solo de un nivel envidiable de dotación en infraestructuras fijas y móviles, sino también de las estructuras de gobernanza (SESAD), ejecución (Red.es) y diagnóstico (ONTSI) para lograr el éxito de la futura estrategia del Gobierno.

En definitiva, España dispone de una de las estructuras más veteranas pero también dinámicas comparada con otros países de nuestro entorno, Red.es, que, gracias al empuje y confianza de las autoridades de tutela en la Secretaria de Estado para el Avance Digital y del propio Ministerio de Economía y Empresa, puede posicionar a España en lugares aún más destacados, trabajando de manera transversal con todas las Administraciones (tanto Administración General del Estado como comunidades autónomas y entidades locales), empresas, emprendedores y ciudadanos, que piensan, como Red.es, que la revolución digital es un asunto cuyo tiempo ha llegado y que reclama a todos estar a la altura de los tiempos.

\section{Bibliografía}

[1] Alba, A. (2016). La Transformación Digital va de Personas. World Economic Forum, Ginebra. https://www.weforum.org/es/agenda/2016/11/ la-transformacion-digital-va-de-personas/

[2] Breznitz, D. y Ornston, D. (2013). The Revolutionary Power of Peripheral Agencies: Explaining Radical Policy Innovation in Finland and Israel. Comparative Political Studies, 46(10), 1219-1245. Disponible en: http://cps. sagepub.com/cgi/content/abstract/46/10/ 1219 ?rss $=1$

[3] Case, S. (2016). La Tercera Ola: el futuro de Internet según uno de sus maximos impulsores. Barcelona: Empresa Activa.

[4] Chesbrough, H. (2012). Open Innovation: Where We've Been and Where We're Going. Research-Technology Management, 55 (August), 20-27.
[5] Clarysse, B.; Wright, M. y Hove, J. V. (2015). A Look Inside Accelerators: building businesses. London. Disponible en: http://www.nesta. org.uk/publications/look-inside-accelerators

[6] Daglio, M.; Gerson, D. y Kitchen, H. (2014, November). «Building organisational capacity for public sector innovation». In Background Paper prepared for the OECD Conference Innovating the Public Sector: from Ideas to Impact, Paris.

[7] Engel, J. S. y Del-Palacio, I. (2009). Global networks of clusters of innovation: Accelerating the innovation process. Business Horizons, 52(5), 493-503. Disponible en: http://dx.doi. org/10.1016/j.bushor.2009.06.001

[8] Hughes, A.; Farren-Handford, M. y Baker, C. (2009). Public Sector Innovation Index: Exploratory Project. Londres: Ernst \& Young. https://www.nesta.org.uk/sites/default/files/ kcfinder/files/Public $\% 20$ Sector $\% 20$ Innovation $\% 20$ Index\%20Exploratory $\% 20$ Project

[9] Insight Foresight Institute (2016). (Re)searching the critical «meso» level: learning innovation agencies and entrepreneurial ecosystems. Disponible en: http://if-institute.org/ agencies-and-ecosystems [Fecha de consulta: 01/03/2016].

[10] Isenberg, D. (2011). The Entrepreneurship Ecosystem Strategy as a New Paradigm for Economic Policy: Principles for Cultivating Entrepreneurships.

[11] Lepori, B. (2011). Coordination modes in public funding systems. Research Policy, 40(3), 355-367. Disponible en: http://www.sciencedirect.com/science/article/pii/S004873331 0002350

[12] Leyden, D. P. y Link, A. N. (2015). Public Sector Entrepreneurship: U.S. Technology and Innovation Policy. Oxford University Press.

[13] Mason, C. y Brown, R. (2014). Entrepreneurial ecosystems and growth oriented entrepreneurship. Final Report to OECD, Paris, 30(1), 77-102.

[14] Marcet, X. (2015). La cultura de la transformación, rompiendo los moldes del Viejo management. Sintetia. Disponible en: https://www.sintetia.com/la-cultura-de-la-transformacionrompiendo-los-moldes-del-viejo-management/ 
[15] Mazzucato, M. (2013). El Estado emprendedor: Mitos del sector público frente al privado. Barcelona: RBA.

[16] O'Hern, M. S. y Rindfleisch, A. (2010). Customer Co-Creation: A Typology and Research Agenda. Review of Marketing Research, 6, Naresh K. Malholtra, ed. Armonk, NY: M.E. Sharpe, 84-106.
[17] Ramaswamy V. y Gouillart, F. (2010). The Power of Co-creation: Build it with them to boost growth, productivity and growth. Free Press, 2010.

[18] Westerman, G.etal. (2011). Digital Transformation: A Road-Map for Billion-Dollar Organizations. MIT Center for Digital Business and Capgemini Consulting, 1-68. 